\title{
Lithium destruction induced by planetary accretion in solar-type stars
}

\author{
Sylvie Théado ${ }^{1}$, Elise Bohuon ${ }^{1}$, and Sylvie Vauclair ${ }^{1}$ \\ ${ }^{1}$ Laboratoire d'Astrophysique de Toulouse et Tarbes, CNRS, Université de Toulouse ; 14 \\ avenue Edouard Belin, 31400 Toulouse, France \\ email: sylvie.vauclair@ast.obs-mip.fr
}

\begin{abstract}
Accretion of planetary (metal-rich) material onto a star in its early phases can produce episodes of thermohaline convection below the outer convective zone. These extramixing phases lead to rapid lithium destruction. The observed dispersion of lithium abundances in solar-type stars can be related to such events.
\end{abstract}

Keywords. Stars: abundances

Lithium depletion in solar-type stars remains a challenge for stellar models. Extramixing below the outer convective zone is needed to explain the observed abundances in the Sun, the solar analogs and the solar twins (e.g., Do Nascimento et al. 2009). Several processes have been invoked in the past to account for this lithium depletion, like rotational induced mixing, but they fail to account for all the observed features and observed abundance dispersion. Meanwhile recent detailed observations of heavy elements in the Sun and solar-type stars show systematic differences, which lead to the idea that accretion may play an important role in their early phases (e.g. Meléndez et al. 2009).

Although no precise statistics can yet be given, it seems possible that many solar-type stars were born with disks, some of them, but not all, leading to observable planets. According to the parameters of the disk and of the central star, matter with abundances different from those of the star can be accreted onto it. It may be either disk gaseous
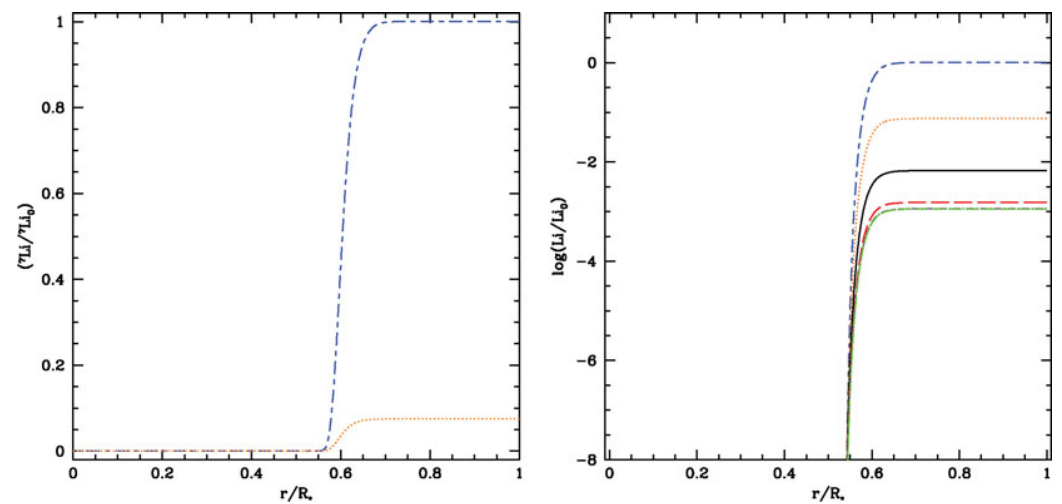

Figure 1. Lithium profiles in $1.10 \mathrm{M}_{\odot}$ stars with planetary accretion. The left panel displays the ratio of the lithium mass fraction to its initial value at 2 Myrs (evolution with atomic diffusion, blue line) and 4 Myrs (after the first accretion episode of $0.03 \mathrm{M}_{j u p}$, orange line). The right panel presents the same ratio in logarithmic units at 2 Myrs (blue line) and after each accretion episode (at 4, 6, 8, 10 and 12 Myrs). Thermohaline mixing strongly decreases the lithium surface abundance. However the depletion saturates after 4 or 5 events. This occurs when the accreted amount of lithium becomes comparable to the depleted one. 

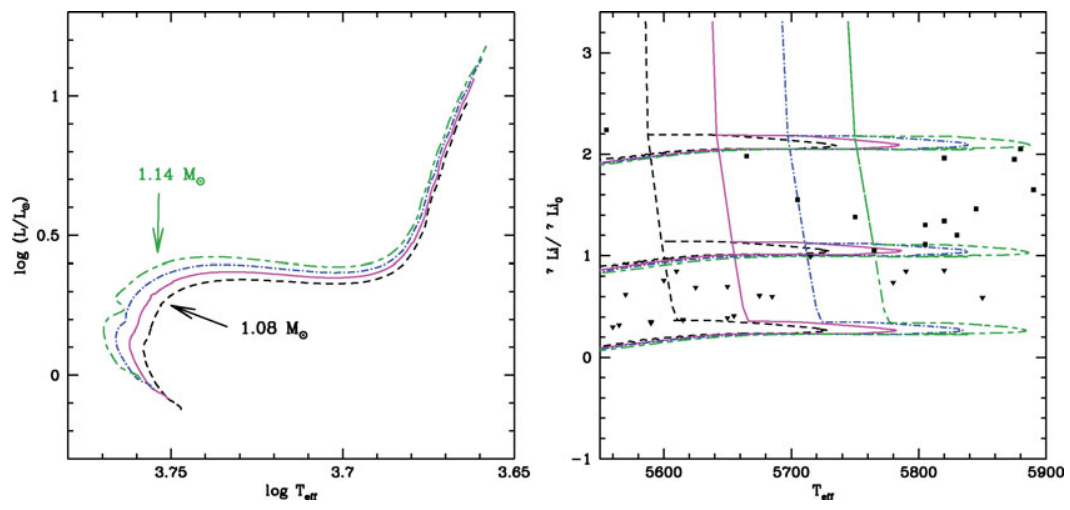

Figure 2. Lithium destruction in stars of various masses : 1.08 (dashed line), 1.10 (solid line), 1.12 (dashed-dotted line) and $1.14 \mathrm{M}_{\odot}$ (long dashed-dashed line) models. The left panel displays the evolutionary tracks. The right panel shows the lithium abundance variations along the evolutionary tracks, as a function of the effective temperature for the same models, including either one, two, or three accretion events of $0.03 \mathrm{M}_{J u p}$ each. The points correspond to the lithium observations by Israelian et al. (2004).

matter (metal-poor) or matter already condensed in the form of planetesimals or planets (metal-rich). The idea that such metal rich-accretion could be the reason for the observed metal overabundances in exoplanet-host stars has now been ruled out (e.g. Vauclair et al. 2008). However, such accretion processes can happen, even if it does not lead to observable metal enrichment, and it may have important consequences on the lithium abundances.

Accretion of planetary matter onto a star in its early phases leads to mean molecular weight inversion below the outer convective zone, unstable against thermohaline convection (e.g. Vauclair 2004). Every time the star accretes planetary material, extra mixing induced by this hydrodynamical instability leads to lithium destruction. We first present computations for a $1.10 \mathrm{M}_{\odot}$ star with $[\mathrm{Fe} / \mathrm{H}]=0.20$ suffering 1 to 5 accretion events of $0.03 \mathrm{M}_{J u p}$ beginning at 2 Myrs and occurring every 2 Myrs afterwards. Then we show examples of other stellar masses and other accreted masses. In all these computations, thermohaline mixing is computed as described in Théado et al. (2009a). Clearly such events can completely modify the lithium abundance and its subsequent evolution. More detailed computations will be given in Théado et al. (2009b).

\section{References}

Do Nascimento, J. D., Castro, M., Meléndez, J., Bazot, M., Théado, S., Porto de Mello, G. F., \& de Medeiros, J. R. 2009, A\& A, 501, 687

Israelian, G., Santos, N. C., Mayor, M., \& Rebolo, R. 2004, A\&A, 414, 601

Meléndez, J., Asplund, M., Gustaffson, B., \& Yong, D. 2009, ApJ (Letters), 704, 66

Théado, S., Vauclair, S., Alecian, G., \& LeBlanc, F. 2009a , ApJ, 704, 1262

Théado, S., Bohuon, E., \& Vauclair, S. 2009b, in preparation

Vauclair, S. 2004, ApJ, 605, 874

Vauclair, S., Laymand, M., Bouchy, F., Vauclair, G., Hui Bon Hoa, A., Charpinet, S. \& Bazot, M. 2008, A\&A (Letters), 482, 5 